\title{
RELATIVE REPRODUCING KERNEL HILBERT SPACES
}

\author{
DANIEL ALPAY, PALLE JORGENSEN, AND DAN VOLOK \\ (Communicated by Pamela B. Gorkin)
}

\begin{abstract}
We introduce a reproducing kernel structure for Hilbert spaces of functions where differences of point evaluations are bounded. The associated reproducing kernels are characterized in terms of conditionally negative functions.
\end{abstract}

\section{INTRODUCTION}

The study of positive definite kernels, and the associated Hilbert spaces, plays a key role in both pure and applied mathematics. It is known (since [2]) that every Hilbert space $\mathscr{H}$ of functions on some set $X$ with the property that point evaluations at points in $X$ are continuous in the norm of $\mathscr{H}$ can be represented by a positive definite kernel. These Hilbert spaces are called reproducing kernel Hilbert spaces. Areas of pure mathematics where reproducing kernel Hilbert spaces play an important role include harmonic analysis [5], representation theory, partial differential equations [7,11] and operator theory [3]. Positive definite kernels also serve as tools in numerical analysis and approximation theory; see e.g. 44. Unfortunately, a consequence of the axiom of choice is that there exist Hilbert spaces of functions which are not reproducing kernel Hilbert spaces, whereas if one accepts instead the weakened axiom of choice, all everywhere defined linear operators between Hilbert spaces are bounded (see [12]) and hence all Hilbert spaces of functions are reproducing kernel Hilbert spaces. See [1,6] for a discussion.

In the present paper our main concern is Hilbert spaces where differences of point evaluations $f \mapsto f(x)-f(y)$ are bounded functionals. This is a wider class than that of reproducing kernel Hilbert spaces. Such spaces are called relative reproducing kernel Hilbert spaces. See Definition 2.1 below. On the applied side, our interest is motivated by electrical network models, where the differences may represent voltage drops, and the absolute value of the voltage function is often irrelevant. In these cases, the Hilbert spaces of relevance are energy spaces, i.e., Hilbert spaces defined from quadratic variations, for example the square sum over all voltage drops across edges in an electric network (see [8, [9] and [10]). When the network is $\mathbb{Z}^{d}$, such spaces always contain dipoles (which correspond to differences of point evaluations), while monopoles (which correspond to evaluations at a single point) appear only

Received by the editors November 7, 2012 and, in revised form, December 6, 2012.

2010 Mathematics Subject Classification. Primary 46E22, 47B32, 42A82.

Key words and phrases. Reproducing kernels, conditionally negative functions, unbounded operators.

The first author thanks the Earl Katz family for endowing the chair which supported his research. The research of the authors was supported in part by the Binational Science Foundation grant No. 2010117. 
for $d \geq 3$. This dichotomy corresponds to a well-known phenomenon in transient random walks on $\mathbb{Z}^{d}$; see [10, Remark 2.11 and Theorem 13.5] and references therein.

The kernels of relative reproducing kernel Hilbert spaces are characterized in terms of conditional negativity rather than definite positivity. The notion of conditional negativity plays a role in infinitely divisible distributions. For example, Schoenberg's theorem about semigroups of positive definite kernels $K_{t}$ states that semigroups of positive kernels admit a tangent space representation; i.e., every semigroup of positive definite kernels $K_{t}$ is associated uniquely to a conditional negative function $\psi$, via the formula $K_{t}=\exp (-t \psi)$. For an application of the latter to $E$-semigroups of endomorphisms in $B(\mathscr{H})$, see 3 .

The paper is structured as follows. In Section 2 the definition of a relative reproducing kernel is introduced and the connections between relative reproducing kernel Hilbert space, reproducing kernel Hilbert space, and continuity of point evaluations are explored. In Section 3 the relation between relative reproducing kernels and conditionally negative functions is established. The last section contains an example (constructed using the axiom of choice) of a relative reproducing kernel Hilbert space which is not a reproducing kernel Hilbert space.

\section{Relative Reproducing Kernel Hilbert sPaCeS}

In this section the Hilbert spaces are over the complex numbers. Note that in Section 3 we consider real Hilbert spaces.

Definition 2.1. Let $\mathscr{H}$ be a Hilbert space of functions defined on the set $X$. We say that it is a relative reproducing kernel Hilbert space if there exists a function $M_{x, y}$ from $X \times X$ into $\mathscr{H}$ such that

$$
F(x)-F(y)=\left\langle F, M_{x, y}\right\rangle_{\mathscr{H}},
$$

for all choices of $x, y \in X$ and of $F \in \mathscr{H}$.

By Riesz' theorem, (2.1) holds if and only if the functional $F \mapsto F(x)-F(y)$ is continuous.

Proposition 2.2. The function $M_{x, y}$ in (2.1) is unique and satisfies

$$
M_{x, y}(t)+M_{y, z}(t)=M_{x, z}(t), \quad \forall x, y, z, t \in X .
$$

Proof. The uniqueness of the function $M_{x y}$ follows from Riesz' representation theorem. This uniqueness and the equality

$$
f(x)-f(z)=f(x)-f(y)+f(y)-f(z)
$$

imply (2.2).

We will call $M_{x, y}$ the relative reproducing kernel.

Proposition 2.3. Let $\mathscr{H}$ be a relative reproducing kernel Hilbert space of functions defined on the set $X$, with relative reproducing kernel $M_{x, y}$. Then the orthogonal complement of the functions $M_{x, y}, x, y \in X$ is equal to the space of constant functions in $\mathscr{H}$. In particular the linear span of the functions $M_{x, y}, x, y \in X$ is dense in $\mathscr{H}$ if and only if $\mathscr{H}$ contains no non-zero constant functions.

Proof. Let $F \in \mathscr{H}$ be orthogonal to all the functions $M_{x, y}$. By (2.1) we have

$$
F(x)=F(y), \quad \forall x, y \in X,
$$

and hence $F$ is constant. 
We note that (2.2) implies that a relative reproducing kernel can always be written as a difference of a given function $T$ from $X$ into $\mathcal{H}$. More precisely, fix any $z_{0} \in X$. Then,

$$
M_{x, y}=T_{x}-T_{y}
$$

where $T_{x}=M_{x, z_{0}}$.

A reproducing kernel Hilbert space is a relative reproducing kernel Hilbert space. It suffices to take

$$
M_{x, y}=K_{x}-K_{y},
$$

where $K_{x}$ is the reproducing kernel, but there are relative reproducing kernel Hilbert spaces which are not reproducing kernel Hilbert spaces.

Proposition 2.4. A Hilbert space $\mathscr{H}$ of functions on the set $X$ is a relative reproducing kernel Hilbert space if and only if there exists a function $h_{x}: X \mapsto \mathscr{H}$ and an everywhere defined possibly unbounded linear functional $C: \mathscr{H} \mapsto \mathbb{C}$ such that

$$
F(x)=\left\langle F, h_{x}\right\rangle_{\mathscr{H}}+C(F), \quad \forall F \in \mathscr{H}, \quad \forall x \in X .
$$

Proof. One direction is clear. Take (2.3) for $x, y \in X$ and subtract. One obtains (2.1) with $M_{x, y}=h_{x}-h_{y}$. Conversely take any $x_{0} \in X$. Then, (2.1) with $y=x_{0}$ implies (2.3) with $h_{x}=M_{x, x_{0}}$ and $C F=F\left(x_{0}\right)$.

See Section 4 for an example. As a corollary of Proposition 2.4, we have:

Corollary 2.5. Let $\mathscr{H}$ be a relative reproducing kernel Hilbert space of functions defined on the set $X$, and assume that for one point $x_{0} \in X$ the point evaluation $F \mapsto F\left(x_{0}\right)$ is bounded. Then, $\mathscr{H}$ is a reproducing kernel Hilbert space.

Proof. It suffices to write for $x \in X$,

$$
F(x)=\left(F(x)-F\left(x_{0}\right)\right)+F\left(x_{0}\right) .
$$

\section{Relative REPRODUCING KERNELS AND NEGATIVE FUNCTIONS}

We first recall that the function $\psi: X \times X \mapsto \mathbb{R}$ is called conditionally negative definite if:

(1) $\psi(x, x)=0$ for all $x \in X$;

(2) $\psi(x, y)=\psi(y, x)$ for all $x, y \in X$;

(3) $\sum_{\ell, j=1}^{n} c_{\ell} c_{j} \psi\left(x_{\ell}, x_{j}\right) \leq 0$ for all $n \in \mathbb{N}$, all $x_{1}, \ldots, x_{n} \in X$ and all numbers $c_{1}, \ldots, c_{n} \in \mathbb{R}$ subject to $\sum_{\ell=1}^{n} c_{\ell}=0$.

Theorem 3.1. Let $\mathscr{H}$ be a relative reproducing kernel real Hilbert space of functions with relative reproducing kernel $M_{x, y}$. Then the real-valued function

$$
\psi(x, y):=\left\|M_{x, y}\right\|_{\mathscr{H}}^{2}=M_{x, y}(x)+M_{y, x}(y)
$$

is conditionally negative. 
Proof. Fix $x_{0}$ and write $M_{x, y}=T_{x}-T_{y}$, where $T_{z}=M_{z, x_{0}}$. Then

$$
\left\|M_{x, y}\right\|^{2}=\left\|T_{x}\right\|^{2}+\left\|T_{y}\right\|^{2}-2\left\langle T_{x}, T_{y}\right\rangle .
$$

From this the properties (1) and (2) of $\psi(x, y)$ are clear.

Now let $n \in \mathbb{N}, x_{1}, \ldots, x_{n} \in X$ and $c_{1}, \ldots, c_{n} \in \mathbb{R}$ be such that

$$
\sum_{\ell=1}^{n} c_{\ell}=0 \text {. }
$$

Then

$$
\begin{aligned}
\sum_{\ell, j=1}^{n} c_{\ell} c_{j}\left\|M_{x_{\ell}, x_{j}}\right\|^{2} & =\sum_{\ell, j=1}^{n} c_{\ell} c_{j}\left(\left\|T_{x_{\ell}}\right\|^{2}+\left\|T_{x_{j}}\right\|^{2}\right)-2 \sum_{\ell, j=1}^{n} c_{\ell} c_{j}\left\langle T_{x_{\ell}}, T_{x_{j}}\right\rangle \\
& =-2\left\|\sum_{\ell=1}^{n} c_{\ell} T_{x_{\ell}}\right\|^{2} \leq 0 .
\end{aligned}
$$

Proposition 3.2. Let $\mathscr{H}$ be a relative reproducing kernel real Hilbert space of functions with relative reproducing kernel $M_{x, y}$. Then for every triple of points $x, y, z \in X$ it holds that

$$
M_{x, y}(z)+M_{y, z}(x)+M_{z, x}(y)=0 .
$$

Proof.

$$
\begin{aligned}
M_{x, y}(z)+M_{y, z}(x)+M_{z, x}(y)=- & M_{y, z}(z)-M_{z, x}(z)+M_{y, z}(x)+M_{z, x}(y) \\
& =\left\langle M_{y, z}, M_{x, z}\right\rangle_{\mathscr{H}}+\left\langle M_{z, x}, M_{y, z}\right\rangle_{\mathscr{H}}=0 .
\end{aligned}
$$

Theorem 3.3. Let $M_{x, y}(z)$ be a function in $X$, satisfying for all $x, y, z, t$ the identities

$$
\begin{aligned}
M_{x, y}(t)+M_{y, z}(t)+M_{z, x}(t) & =0 \\
M_{x, y}(z)+M_{y, z}(x)+M_{z, x}(y) & =0 .
\end{aligned}
$$

Suppose that the function

$$
\psi(x, y):=M_{x, y}(x)+M_{y, x}(y)
$$

is conditionally negative. Then there is a real Hilbert space which has $M_{x, y}$ as its relative reproducing kernel.

Proof. Fix $x_{0}$ and denote $T_{x}=M_{x, x_{0}}$. By (3.1) with $x=y=z$ we get

$$
M_{z, z}(t)=0 \text {. }
$$

Again by (3.1), now with $y=z$, we get

$$
M_{x, y}(t)=-M_{y, x}(t) .
$$

Once more by (3.1) with $z=x_{0}$ we have

$$
M_{x, y}(t)=-M_{y, x_{0}}(t)-M_{x_{0}, x}(t)=M_{x, x_{0}}(t)-M_{y, x_{0}}(t)=T_{x}(t)-T_{y}(t),
$$

and it follows that

$$
\psi(x, y)=T_{x}(x)-T_{x}(y)-T_{y}(x)+T_{y}(y) .
$$


As in the proof of [5, Proposition 3.2 p. 82], we consider the function

$$
\phi(x, y)=\frac{1}{2}\left(\psi\left(x, x_{0}\right)+\psi\left(x_{0}, y\right)-\psi(x, y)\right) .
$$

We claim that $\phi(x, y)$ is positive in $X$. Indeed, since $\psi(x, y)=\psi(y, x), \phi(x, y)=$ $\phi(y, x)$. Let $x_{1}, \ldots, x_{n} \in X$ and $c_{1}, \ldots, c_{n} \in \mathbb{R}$. Denote

$$
c_{0}=-\sum_{j=1}^{n} c_{j} .
$$

Then

$$
\begin{aligned}
0 \geq \sum_{j, \ell=0}^{n} c_{\ell} c_{j} \psi\left(x_{j}, x_{\ell}\right)=\sum_{j, \ell=1}^{n} c_{\ell} c_{j} \psi\left(x_{j}, x_{\ell}\right)+2 c_{0} \sum_{j=1}^{n} c_{j} \psi\left(x_{0}, x_{j}\right) & \\
& =-\sum_{j, \ell=1}^{n} c_{\ell} c_{j} \phi\left(x_{j}, x_{\ell}\right) .
\end{aligned}
$$

Next we observe that, as follows from (3.3),

$$
\phi(x, y)=\frac{1}{2}\left(T_{x}(y)+T_{y}(x)-T_{x}\left(x_{0}\right)-T_{y}\left(x_{0}\right)\right) .
$$

Set $z=x_{0}$ in (3.2) to obtain

$$
T_{x}\left(x_{0}\right)-T_{y}\left(x_{0}\right)+T_{y}(x)-T_{x}(y)=0,
$$

which implies

$$
\phi(x, y)=T_{x}(y)-T_{x}\left(x_{0}\right) .
$$

Finally, consider the Hilbert space $\mathcal{H}$ obtained as a completion of the linear span (over $\mathbb{R}$ ) of the functions $T_{x}$ with respect to the inner product

$$
\left\langle T_{x}, T_{y}\right\rangle_{\mathcal{H}}=\phi(x, y) \text {. }
$$

For $x, y, z \in X$ we have

$$
\left\langle T_{x}, M_{y, z}\right\rangle=\left\langle T_{x}, T_{y}\right\rangle-\left\langle T_{x}, T_{z}\right\rangle=\phi(x, y)-\phi(x, z)=T_{x}(y)-T_{x}(z) .
$$

Since the span of $T_{x}$ is dense in $\mathcal{H}, \mathcal{H}$ is a real relative reproducing kernel Hilbert space.

Corollary 3.4. Let $\psi(x, y)$ be a conditionally negative function. Then there exists a real relative reproducing kernel Hilbert space $\mathscr{H}$ of functions with relative reproducing kernel

$$
M_{x, y}(z):=\frac{\psi(z, y)-\psi(z, x)}{2}
$$

which satisfies

$$
\left\|M_{x, y}\right\|_{\mathscr{H}}^{2}=\psi(x, y)
$$

Proof. It is easily checked that $M_{x, y}(z)$ satisfies (3.1) and (3.2). Furthermore,

$$
M_{x, y}(x)+M_{y, x}(y)=\frac{\psi(x, y)+\psi(y, x)-\psi(x, x)-\psi(y, y)}{2}=\psi(x, y) .
$$




\section{An example of a Relative Reproducing KERNEL HILBERT SPACE}

We now give an example of a relative reproducing kernel space which is not a reproducing kernel Hilbert space. Let $\mathcal{H}$ be a Hilbert space of functions on a set $X$, which do not contain the constants (for example the Hardy space of the open upper half-plane). Let $C$ be an everywhere defined unbounded linear operator with complex values, and let $\left(h_{x}\right)_{x \in X}$ be a family of functions in $\mathcal{H}$ indexed by $X$, and such that the linear span of the functions $h_{x}-h_{y}, x, y \in X$ is dense in $\mathcal{H}$. We consider functions of the form

$$
(\mathbf{I}(f))(x)=\left\langle f, h_{x}\right\rangle_{\mathcal{H}}+C(f), \quad f \in \mathcal{H} .
$$

Then:

Lemma 4.1.

$$
(\mathbf{I}(f)) \equiv 0 \quad \Longleftrightarrow f=0
$$

Proof. We have

$$
\left\langle f, h_{x}\right\rangle_{\mathcal{H}} \equiv-C(f),
$$

and so

$$
\left\langle f, h_{x}-h_{y}\right\rangle_{\mathcal{H}} \equiv 0,
$$

and so $f=0$ by the hypothesis on the $h_{x}$ and since $\mathcal{H}$ has no non-zero constant function.

Theorem 4.2. The linear space $\mathscr{H}$ of functions of the form $\mathbf{I}(f)$ with the inner product

$$
\langle\mathbf{I}(f), \mathbf{I}(g)\rangle_{\mathscr{H}}=\langle f, g\rangle_{\mathcal{H}}
$$

is a relative reproducing kernel space which is not a reproducing kernel space.

Proof. We first note that $\mathscr{H}$ is a Hilbert space since $\mathbf{I}$ is one-to-one and the inner product on $\mathscr{H}$ is given via (4.1). Suppose that $\mathscr{H}$ is a reproducing kernel Hilbert space. Then by Riesz theorem, for $x \in X$ there exists $g_{x} \in \mathcal{H}$ such that, for all $f \in \mathcal{H}$,

$$
(\mathbf{I}(f))(x)=\left\langle\mathbf{I}(f), \mathbf{I}\left(g_{x}\right)\right\rangle_{\mathscr{H}}=\left\langle f, g_{x}\right\rangle_{\mathcal{H}}
$$

so that

$$
C(f)=\left\langle f, g_{x}\right\rangle_{\mathcal{H}}-\left\langle f, h_{x}\right\rangle_{\mathcal{H}} .
$$

Since $C$ has been assumed to be unbounded, this gives a contradiction.

To verify that $\mathscr{H}$ is a relative reproducing kernel Hilbert space, we write for $f \in \mathcal{H}$ and $x, y \in X$ :

$$
\begin{aligned}
(\mathbf{I}(f))(x)-(\mathbf{I}(f))(y) & =\left\langle f, h_{x}-h_{y}\right\rangle_{\mathcal{H}} \\
& =\left\langle\mathbf{I}(f), M_{x, y}\right\rangle_{\mathscr{H}},
\end{aligned}
$$

where

$$
M_{x, y}=\mathbf{I}\left(h_{x}\right)-\mathbf{I}\left(h_{y}\right) .
$$

We remark that by Corollary 2.5 the example gives a Hilbert space where no point evaluation is bounded. 


\section{REFERENCES}

[1] D. Alpay and T. M. Mills, A family of Hilbert spaces which are not reproducing kernel Hilbert spaces, J. Anal. Appl. 1 (2003), no. 2, 107-111. MR.1986785 (2004d:46032)

[2] N. Aronszajn, Theory of reproducing kernels, Trans. Amer. Math. Soc. 68 (1950), 337-404. $\operatorname{MR} 0051437(14,479 \mathrm{c})$

[3] William Arveson, An addition formula for the index of semigroups of endomorphisms of $B(H)$, Pacific J. Math. 137 (1989), no. 1, 19-36. MR.983326 (90c:47074)

[4] Kendall Atkinson and Weimin Han, Spherical harmonics and approximations on the unit sphere: an introduction, Lecture Notes in Mathematics, vol. 2044, Springer, Heidelberg, 2012. MR2934227

[5] Christian Berg, Jens Peter Reus Christensen, and Paul Ressel, Harmonic analysis on semigroups, Theory of positive definite and related functions. Graduate Texts in Mathematics, vol. 100, Springer-Verlag, New York, 1984. MR747302 (86b:43001)

[6] W. Donoghue and P. Masani, A class of invalid assertions concerning function Hilbert spaces, Bol. Soc. Mat. Mexicana (2) 28 (1983), no. 2, 77-80. MR791090 (87i:46054)

[7] Peter Giesl and Holger Wendland, Approximating the basin of attraction of time-periodic ODEs by meshless collocation of a Cauchy problem, Discrete Contin. Dyn. Syst. (2009), 259268. Dynamical Systems, Differential Equations and Applications. 7th AIMS Conference, suppl. MR,2641402 (2011d:37159)

[8] Palle E. T. Jorgensen and Erin P. J. Pearse, A discrete Gauss-Green identity for unbounded Laplace operators and the transience of random walks. Israel J. Math. 196 (2013), no. 1, 113-160. MR3096586

[9] Palle E. T. Jorgensen and Erin Peter James Pearse, A Hilbert space approach to effective resistance metric, Complex Anal. Oper. Theory 4 (2010), no. 4, 975-1013, DOI 10.1007/s11785-009-0041-1. MR2735315 (2011j:05338)

[10] Palle E. T. Jorgensen and Erin Peter James Pearse. Operator theory of electrical resistance networks, arXiv:0806.3881, November 2009.

[11] Holger Wendland, Scattered data approximation, Cambridge Monographs on Applied and Computational Mathematics, vol. 17, Cambridge University Press, Cambridge, 2005. MR2131724(2006i:41002)

[12] J. D. Maitland Wright, All operators on a Hilbert space are bounded, Bull. Amer. Math. Soc. 79 (1973), 1247-1250. MR0328649 (48 \#6991)

Department of Mathematics, Ben Gurion University of the Negev, P.O.B. 653, Be'er SHEVA 84105, ISRAEL

E-mail address: dany@math.bgu.ac.il

Department of Mathematics, 14 MLH, The University of Iowa, Iowa City, Iowa 522421419

E-mail address: jorgen@math.uiowa.edu

Department of Mathematics, 138 Cardwell Hall, Kansas State University, ManhatTAN, KANSAS 66506

E-mail address: danvolok@math.ksu.edu 